\title{
Altered neonatal cord blood oxylipidome in association with exposure to particulate matter in the early life environment
}

\author{
Dries Martens ${ }^{1 *}$, Sandra Gouveia-Figueira ${ }^{2}$, Narjes Madhloum³ ${ }^{3}$ Bram G Janssen ${ }^{3}$, Michelle Plusquin ${ }^{3}$, \\ Bertil Forsberg ${ }^{4}$, Malin L Nording ${ }^{3}$, Tim S Nawrot ${ }^{3}$ \\ From Methods in Epidemiology Symposium \\ Leuven, Belgium. 17 September 2015
}

\section{Background and aims}

As part of the lipidome, oxylipins are bioactive lipid compounds originating from oxidation of different fatty acids. We studied oxylipin profiles in cord blood as part of the lipidome in early life in association with in utero exposure to particulate air pollution.

\section{Methods}

Oxylipins were extracted from 197 cord blood plasma samples from the ENVIRONAGE (ENVIRonmental influence $O N$ AGEing in early life) birth cohort in Belgium. Thirty-seven specific oxylipins reflecting the cyclooxygenase (COX), lipoxygenase (5-LOX and 12/15LOX) and cytochrome P450 (CYP) metabolic pathways were quantified by ultra-performance liquid chromatography coupled to electrospray ionization tandem mass spectrometry (UPLC-ESI-MS/MS). Principal component analysis and multiple regression models were applied to associate oxylipin pathways as well as individual metabolites with in utero $\mathrm{PM}_{2.5}$ exposure, while adjusting for newborns gender, gestational duration, maternal age, maternal smoking status, maternal BMI, and cord blood total cholesterol and HDL levels.

\section{Results}

$\mathrm{PM}_{2.5}$ exposure during pregnancy averaged (25th-75th percentile) $15.7(13.5-17.5) \mu \mathrm{g} / \mathrm{m}^{3}$. Six metabolites combined in a principal component (PC), representing the 5-LOX pathway was positively associated with $\mathrm{PM}_{2.5}$ exposure during the entire $(?=0.11 ; 95 \% \mathrm{CI}: 0.03,0.20$; $\mathrm{p}=0.01)$ and second trimester of pregnancy $(\beta=0.06$;
95\% CI: 0.01, 0.10; $\mathrm{p}=0.01$ ). Eleven metabolites combined in a PC representing the 12/15-LOX pathway was positively associated with $\mathrm{PM}_{2.5}$ during the second trimester of pregnancy ( $\beta=0.09 ; 95 \%$ CI: $0.03,0.15 ; \mathrm{p}=$ 0.006). No associations were found for the COX and CYP pathways.

\section{Conclusions}

In utero exposure to particulate matter was associated with the lipoxygenase pathways (5-LOX and 15-LOX) in newborns. These changes at the level of the lipidome indicate an altered inflammatory state of the newborn at birth induced by air pollution during in utero life.

\section{Authors' details}

${ }^{1}$ Hasselt University, Diepenbeek, Belgium. ${ }^{2}$ Department of Chemistry, Umeå University, Umeå, Sweden. ${ }^{3}$ Centre for Environmental Sciences, Hasselt University, Diepenbeek, Belgium. ${ }^{4}$ Division of Occupational and Environmental Medicine, Umeå University, Umeå, Sweden.

Published: 17 September 2015

doi:10.1186/2049-3258-73-S1-O5

Cite this article as: Martens et al:: Altered neonatal cord blood oxylipidome in association with exposure to particulate matter in the early life environment. Archives of Public Health 2015 73(Suppl 1):O5.

${ }^{1}$ Hasselt University, Diepenbeek, Belgium

Full list of author information is available at the end of the article 